\title{
Embodiment of Abstract Concepts: Good and Bad in Right- and Left-Handers
}

\author{
Daniel Casasanto \\ Stanford University and the Max Planck Institute for Psycholinguistics
}

\begin{abstract}
Do people with different kinds of bodies think differently? According to the body-specificity hypothesis, people who interact with their physical environments in systematically different ways should form correspondingly different mental representations. In a test of this hypothesis, 5 experiments investigated links between handedness and the mental representation of abstract concepts with positive or negative valence (e.g., honesty, sadness, intelligence). Mappings from spatial location to emotional valence differed between rightand left-handed participants. Right-handers tended to associate rightward space with positive ideas and leftward space with negative ideas, but left-handers showed the opposite pattern, associating rightward space with negative ideas and leftward with positive ideas. These contrasting mental metaphors for valence cannot be attributed to linguistic experience, because idioms in English associate good with right but not with left. Rather, right- and left-handers implicitly associated positive valence more strongly with the side of space on which they could act more fluently with their dominant hands. These results support the body-specificity hypothesis and provide evidence for the perceptuomotor basis of even the most abstract ideas.
\end{abstract}

Keywords: abstract concepts, body-specificity hypothesis, embodied cognition, emotional valence, metaphor

According to theories of embodied cognition, thoughts comprise mental simulations of bodily experiences (Barsalou, 1999; Feldman, 2006; Goldstone \& Barsalou, 1998; Lakoff \& Johnson, 1999; Prinz, 2002). If thinking is embodied in this sense, then people with different kinds of bodies must think differently. That is, if concepts and word meanings are constituted in part by simulations of people's own perceptions and actions, then people with different bodily characteristics, who interact with their physical environments in systematically different ways, should form correspondingly different mental representations. I call this proposal the body-specificity hypothesis.

The five experiments reported here tested this hypothesis and sought to overcome two obstacles to advancing embodied theories of mental representation: (1) devising experiments in which embodied theories and their alternatives make contrasting predictions and (2) determining the experiential origins of our mental representations, even in abstract conceptual domains. Framing experimental predictions in terms of body-specificity made it possible to discover a bodily basis for people's abstract mental representations

Daniel Casasanto, Department of Psychology, Stanford University, and Max Planck Institute for Psycholinguistics, Nijmegen, the Netherlands.

A portion of this research was reported at the 30th Annual Conference of the Cognitive Science Society, Washington, DC, July 2008. This research was supported in part by National Research Service Award Postdoctoral Fellowship F32MH072502 and by Spanish Ministry of Education and Science Grant SEJ2006-04732/PSIC, DGI. I am grateful to Mandana Seyfeddinipur, Rachel Staum, Merel van Rees Vellinga, and Josje Verhagen for collecting and coding questionnaire data; to Laura Staum Casasanto and Herb Clark for invaluable discussions; and to Fernanda Ferreira for comments on a draft of the article.

Correspondence concerning this article should be addressed to Daniel Casasanto, Max Planck Institute for Psycholinguistics, P.O. Box 310, 6500 AH Nijmegen, the Netherlands. E-mail: daniel.casasanto@mpi.nl that could not be predicted (or explained) on the basis of patterns in language and culture.

It is easy to imagine ways in which specific features of our bodies might influence our mental representations of concrete objects and actions. For example, if thinking about objects involves mentally simulating their colors (Simmons et al., 2007), then mental representations of apples should be qualitatively different between individuals with red-green color blindness and individuals with normal vision. If thinking about actions involves mentally simulating the way we typically execute them, actions that we perform with our dominant hands such as throwing a ball, turning a key, or signing a check should have different neurocognitive representations in right-handed and left-handed individuals (Casasanto, 2008a; Longcamp, Anton, Roth, \& Velay, 2005; Willems, Hagoort, \& Casasanto, in press).

But how might body-specificity be relevant to the mental representation of more abstract concepts, like goodness and badness, victory and loss, deceit and honesty? Our ability to formulate such abstract ideas presents a challenge to embodied theories of concepts, in general: How can perceptuomotor simulations help to represent things we can never perceive with the senses or act upon with the muscles? Like many abstract concepts, these notions carry either positive or negative emotional valence. Several lines of research have suggested important links between valence and perception and action in physical space.

In English and other languages, metaphorical expressions tend to associate positive and negative valence with the top and bottom of a vertical spatial continuum (Lakoff \& Johnson, 1980, 1999): a happy person is "high on life," but a sad person is "down in the dumps"; a genius is "highly intelligent," but an idiot has a "low IQ"; some research assistants are "top flight," whereas others are at the "bottom of the barrel." According to theories of metaphorical mental representation (e.g., Lakoff \& Johnson, 1999), these linguistic metaphors reflect mental metaphors (Casasanto, 2008b): 
nonlinguistic associative mappings from the concrete source domain of space to relatively abstract target domains with positive or negative valence. Behavioral studies provide evidence that mental metaphors from physical space structure our representations not only of valenced concepts (Casasanto \& Dijkstra, 2009; Meier \& Robinson, 2004) but also of time (Boroditsky, 2000, 2001; Casasanto \& Boroditsky, 2008), number (Dehaene, Bossini, \& Giraux, 1993), emotional attachment (L. E. Williams \& Bargh, 2008), power (Schubert, 2005), and similarity (Casasanto, 2008b) — even when we're not using any linguistic metaphors (cf. Murphy, 1996, 1997).

Mental metaphors provide a potential solution, or at least part of a solution, to the problem of representing abstract ideas via embodied simulations. Thinking about affective states or making affective judgments could involve mental simulations in both source and target domains. Target domain representations could comprise partial reenactments of emotional states in the regions of the brain that give rise to emotional experiences. Simulating affection could involve recapitulating patterns of activity in the nucleus accumbens that produce the interoceptive experience of affection, and simulating fear recapitulating patterns of activity in the amygdala that produce the experience of fear (Damasio, 2001). These primitive target domain representations may be too vague or fleeting to support higher order reasoning about emotional states and may be resistant to the kinds of verbal and imagistic coding that can scaffold such reasoning. Mental metaphors import the inferential structure of source domains like space into target domains, allowing us to envision, measure, and compare the height of people's excitement, the depth of their sadness, or the breadth of their compassion (Boroditsky, 2000; Casasanto, 2008c; Pinker, 1997). To the extent that mental representations in perceptuomotor source domains constitute abstract concepts, these concepts can be instantiated by the same neural and mental structures that simulate perception and action in the physical world.

\section{Are Mental Metaphors Necessarily "Embodied"?}

Considerable evidence for the existence of mental metaphors has accumulated, but their experiential origins have remained unknown. Two proposals have emerged from nearly distinct literatures, the first positing that mental metaphors arise due to correlations in bodily experience and the second due to correlations in linguistic experience. On the first proposal (e.g., Lakoff \& Johnson, 1999; Piaget, 1927/1969), mental metaphors like Positive Is Up and Negative Is Down could be established as people implicitly learn associations between physical experiences and emotional states that typically co-occur (e.g., standing tall when we feel proud, slouching when we feel dejected). Linguistic metaphors then encode preexisting mental metaphors developed on the basis of relationships between different types of bodily experiences (i.e., perceptuomotor experiences in source domains and interoceptive experiences in target domains). Social psychological studies have been interpreted as supporting this proposal (Barsalou, Niedenthal, Barbey, \& Ruppert, 2003). Participants perform better and produce more positive evaluations on various tasks when they assume upright body postures in an experimental setting that are similar to the postures people assume spontaneously when they are feeling good in their everyday lives. In one experiment, participants persisted longer in a puzzle-solving task after assuming an upright posture as opposed to a slouching posture (Riskind \& Gotay,
1982), and in another participants expressed more pride in their test performance after sitting upright during the critical phase of the experiment than after slouching (Stepper \& Strack, 1993).

On the alternative proposal, mental metaphors are established through experience by using linguistic metaphors. Using spatial words in both literal and metaphorical contexts (e.g., a high shelf, a high ideal) could cause structural elements of the concrete source domain to be transferred to abstract target domain representations in the mind of the language learner via analogical processes that are not necessarily "embodied" (see Boroditsky, 2000; Gentner, Bowdle, Wolff, \& Boronat, 2001). One reason to consider this possibility is that associations between valence and vertical space have been shown to generalize beyond concepts that have obvious perceptuomotor correlates. For example, in one experiment, participants were faster to judge words like polite and rude as having positive or negative valence when positive words were presented at the top and negative words at the bottom of a computer screen (Meier \& Robinson, 2004). In another experiment, participants were faster to make lexical decisions on positive-valence words (e.g., brave, ethical, wealthy) when they were presented above nonword distractors, and on negative-valence words (e.g., poverty, failure, hate) when presented below nonword distractors, even though neither the spatial position of the words nor their valence was relevant to the task (Casasanto \& Nabieva, 2007). These results are problematic for a purely embodied theory of mental metaphor: What are the perceptuomotor correlates of politeness, poverty, or ethics that could plausibly link these concepts with vertical space?

Linguistic conventions associating valence with vertical space are reinforced by other nonlinguistic cultural conventions, such as the "thumbs up" and "thumbs down" gestures that indicate approval and disapproval. Once these linguistic and nonlinguistic conventions are part of a culture, they can serve as the basis for metaphorical mappings in the minds of individual learners, obviating the role of direct bodily experience. As such, data that support metaphor theory do not necessarily support embodiment theory. Mental metaphors could be learned from patterns in language and culture. Behavioral effects that reveal the existence of mental metaphors could result from spreading activation between nodes in an amodal conceptual network that are habitually coactivated during language use, rather than resulting from correlated physical and emotional experiences. ${ }^{1}$

\footnotetext{
${ }^{1}$ The proposal that correlations in linguistic experience give rise to Good Is Up mental metaphors in the individual learner raises the question of how linguistic metaphors for valence arose in the first place, and why they are so common across languages. It may be that correlations in direct bodily experience resulted in the construction of these linguistic conventions during the course of linguistic/cultural evolution. Yet, the existence of linguistic metaphors in a culture is not evidence of prelinguistic mental metaphors in the individual. Metaphors in language and other linguistic structures (e.g., words for exact numbers) can precede concepts in the individual learner and lead to the creation of new conceptual links via learning processes that do not need to be grounded directly in perceptuomotor experience (see Carey, 2004; Dehaene, Bossini, \& Giraux, 1993; Gentner et al., 1997). Even if direct bodily experience is necessary on the timescale of biological evolution or cultural/linguistic evolution, it may not be necessary on the timescale of conceptual development in the individual learner (Tomasello, 2003; Vygotsky, 1934/1986).
} 
Is it possible to determine whether mental metaphors arise from correlations in linguistic experience versus correlations in nonlinguistic bodily experience, or from some combination of linguistic and bodily experiences? Because patterns in language closely mirror patterns of bodily interactions with the environment (Clark, 1973), language and bodies generally make the same neural and behavioral predictions (Machery, 2007; Zwaan \& Yaxley, 2003a, 2003b). To overcome this obstacle, the present study tested for a body-specific mapping between space and valence that is not encoded in any known language or cultural artifacts but that was predicted on the basis of particulars of our bodily experience.

\section{When Is the Right Side the "Right" Side?}

In addition to links between valence and vertical space, there are robust associations between valence and the right and left sides of the body. Actions performed with the right side of the body (e.g., contracting the muscles of the right hand or the right side of the face, or viewing stimuli in the right visual hemifield) correlate with positive affect, whereas the same actions performed with the left side of the body correlate with negative affect (e.g., Davidson, 1992; Natale, Gur, \& Gur, 1983). This association of positive and negative valence with the right and left sides of the body may be linked to motivational systems controlling approach and avoidance behaviors. Approach has been lateralized to the left frontal lobe (which controls the right side of the body), and avoidance to the right frontal lobe (which controls the left side of the body; e.g., Davidson, Ekman, Saron, Senulis, \& Friesen, 1990; Schiff \& Bassel, 1996).

The same associations of right with "positive" and left with "negative" that are revealed by neuropsychological tests are also enshrined in everyday linguistic expressions. English idioms like "the right answer" and "my right-hand man" associate good things with rightward space, and complementary idioms like "out in left field" and "two left feet" associate bad things with leftward space. The Latin words for right and left-dexter and sinister-form the roots of English words meaning "skillful" and "evil," respectively. Similar patterns are found across many languages. The words for right in French (droite) and in German (Recht) are closely related to the words meaning "a 'right' or privilege accorded by the law," whereas the words for left in French (gauche) and German (Links) are related to words meaning "distasteful" or "clumsy." Such idioms are evident in nonlinguistic conventions in some cultures, such as Ghanaian society, where pointing and gesturing with one's left hand is prohibited (Kita \& Essegbey, 2001). According to Islamic doctrine, the left hand should be used for dirty jobs, whereas the right hand is used for eating. Likewise, the left foot is used for stepping into the bathroom, and the right foot for entering the mosque.

Why, across cultures and modalities, is left conventionally associated with bad and right with good? This directional preference for valence does not appear to vary with the direction of reading and writing in a culture, which correlates with mappings from horizontal space to other abstract concepts such as number and time. In cultures that use left-to-right writing systems, like Englishspeaking cultures, the mental number line increases from left to right, and likewise the mental time line flows from left to right; the opposite is true in Arabic-speaking cultures, which use right-to-left writing systems (Chatterjee, 2001; Dehaene, Bossini, \& Giraux,
1993; Maas \& Russo, 2003; Tversky, Kugelmass, \& Winter, 1991; Zebian, 2005). Yet, despite these cross-cultural reversals in horizontal mappings for number and time, linguistic and cultural conventions in Arabic-speaking and English-based cultures reveal the same Good Is Right mapping. Valence is apparently insensitive to writing direction. One possible explanation for this seemingly universal directional preference is that it arises from universal properties of the human brain and mind, perhaps related to innate hemispheric specialization for approach and avoidance motivational systems. Once established due to innate neurobiological factors, conventions in language and culture may reinforce this implicit preference for the right.

An alternative possibility, however, is that left-right conventions in language and culture arise as a consequence of bodyspecific associations between space and valence. Bodies are lopsided. Most people have a dominant hand, usually the right hand (Corballis \& Beale, 1976), and therefore interact with their environment more fluently on one side of body-centered space than the other. Greater perceptuomotor fluency has been shown to correlate with more positive evaluations (Oppenheimer, 2008; Reber, Winkielman, \& Schwarz, 1998). For example, expert typists have shown a preference for pairs of letters that can be typed easily over pairs that are more difficult to type (even when typing is not relevant to the task), suggesting that motor experience can influence affective judgments (Beilock \& Holt, 2007; Van den Bergh, Vrana, \& Eelen, 1990). In a sense, we are all "experts" at using our dominant hands. Perhaps over a lifetime of lopsided perceptuomotor experience, people come to implicitly associate good things with the side of space they can interact with more fluently and bad things with the side of space they interact with less fluently? On this body-specific possibility, the apparent universality of the Good Is Right mapping suggested by linguistic and cultural conventions could be a result of right-handers' predominance in the population, worldwide: Linguistic and cultural conventions may develop according to the implicit body-specific mental metaphors of the majority.

Five experiments tested associations between valence and horizontal space in right- and left-handed individuals, to determine whether these mappings are universal or body-specific. If they are universal, either due to invariant properties of the human brain and mind or to pervasive patterns in language and culture, then both right- and left-handers should preferentially associate good with right and bad with left. Alternatively, if they are body-specific, then right- and left-handers should show opposite patterns, each group associating good things more strongly with their dominant side and bad things with their nondominant side.

\section{Experiment 1: Diagramming the Good and the Bad}

Participants performed a pencil-and-paper diagram task in which they drew one animal in each of two boxes, located either to the left and right of a cartoon figure (in the horizontal condition; see Figure 1a) or above and below a cartoon figure (in the vertical condition; see Figure 1b). Instructions indicated that the cartoon figure likes certain animals and thinks they are good but dislikes other animals and thinks they are bad. Participants were instructed to draw a good animal in the box they thought best represented good things and a bad animal in the box that best represented bad things. 


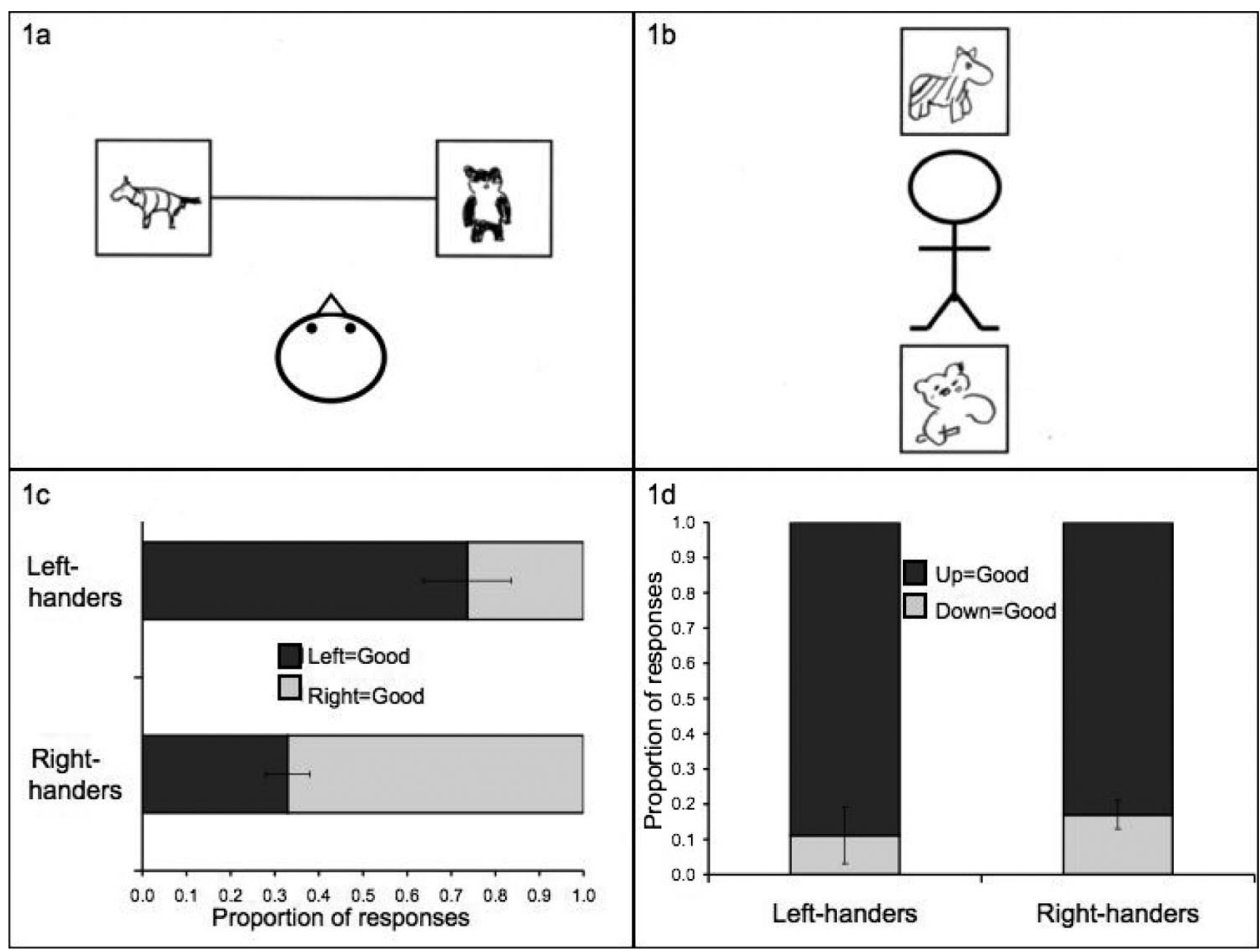

Figure 1. Top: Examples of stimuli and responses from Experiment 1. 1a: Horizontal condition. 1b: Vertical condition. Bottom: Results of Experiment 1. 1c: Proportion of left- and right-handers who drew the good animal in the left box (dark bars) and the right box (light bars). 1d: Proportion of left- and right-handers who drew the good animal in the top box (dark bars) and the bottom box (light bars). Error bars indicate standard error of the mean.

The horizontal condition served as a test of the body-specificity hypothesis: If the horizontal spatialization of valence is bodyspecific, then right- and left-handers should show opposite preferences for the placement of good and bad animals in the left and right boxes. Alternatively, if the horizontal spatialization of valence is universal, then right- and left-handers should show the Good Is Right preference that is suggested by linguistic and cultural conventions.

The vertical condition served as a control. Regardless of handedness, we all use the same linguistic and cultural conventions that associate up with good, and we all experience the same correlations between upright body posture and positive mood. Therefore, both right- and left-handers should tend to place the good animal on top and the bad animal on the bottom.

\section{Method}

Participants. Students from the Stanford University and the University of California, Riverside, communities took part in exchange for payment or course credit $(N=219 ; 28$ left-handers, 191 right-handers by self-report). Participants were randomly assigned to perform either the horizontal task ( $n=104$; 19 lefthanders, 85 right-handers) or the vertical task ( $n=115$; 9 lefthanders, 106 right-handers).

Materials and procedure. A paper-and-pencil diagram task with instructions on one side of a page and responses on the other side was distributed among unrelated questionnaires. Each partic- ipant completed either the horizontal or the vertical task, which differed primarily in the orientation of the boxes; in addition, different cartoon characters were used for the horizontal and vertical conditions. This was necessary because in the horizontal condition it was critical that the cartoon character's spatial perspective be the same as the participants' so that their assignment of animals to the left and right boxes could be interpreted unambiguously, whereas in the vertical condition it was critical that up/ down space be distinguishable from front/back space in the twodimensional diagram (see Figures 1a and 1b).

Instructions indicated that when participants flipped the page they would meet a cartoon character who was planning a trip to the zoo. They were told that the character loves zebras and thinks they are good but hates pandas and thinks they are bad (or vice versa, depending on the version of the questionnaire a participant received). Their task was to draw a zebra in the box that best represents good things like seeing zebras and a panda in the box that best represents bad things like seeing pandas (or vice versa) The assignment of valence to the panda and zebra was counterbalanced across participants. The order in which participants were instructed to draw the good and bad animals was also counterbalanced, to ensure that any associations between space and valence in participants' judgments were not confounded with associations between the side of space and the temporal order in which they drew the animals. After completing the diagram task, participants were asked to report their handedness (i.e., "Are you left-handed or 
right-handed? Circle: L or R") among three other filler questions (e.g., "Are you male or female? Circle: M or F"). The questionnaire took $1-2$ min to complete.

\section{Results and Discussion}

In the horizontal task, a majority (74\%) of left-handers drew the good animal in the box on the left of the cartoon character (sign test on 5 right side vs. 14 left side, $p_{\text {rep }}=0.91$ ), whereas a majority (67\%) of right-handers drew the good animal in the box on the right ( sign test on 57 right side vs. 28 left side, $p_{\text {rep }}=0.99$; see Figure 1c). ${ }^{2}$ By Fisher's exact test, there was a significant correlation between the handedness of the participant and the left-right placement of the good and bad animals ( $p_{\text {rep }}$ for the exact association $=0.99)$. The strength of this association was evaluated with a binary logistic regression. The odds ratio (OR) for the regression of left-right preference on handedness was estimated at $5.70(95 \%$ confidence interval $[\mathrm{CI}]=1.87-17.41)$, indicating that right-handers were nearly six times more likely than left-handers to place the good animal on the right and the bad animal on the left.

Since horizontal space has been associated with spatial and temporal sequences (Clark, 1973; Dehaene, Bossini, \& Giraux, 1993) as well as with valence, an analysis was conducted to evaluate the possibility that the order in which participants drew the animals influenced their placement. Due to counterbalancing, approximately equal numbers of participants were instructed to draw the good animal first (45 right-handers, 10 left-handers) and the bad animal first (40 right-handers, 9 left-handers). When the good animal came first, 69\% of participants placed the good animal on their dominant side. Likewise, when the bad animal came first, $67 \%$ of participants placed the good animal on their dominant side. The proportions of body-specific responses did not differ significantly as a function of the temporal order in which participants drew the good and bad animals $\left(p_{\text {rep }}=0.64\right)$, suggesting that the temporal order of responses was not responsible for the observed effect. There was also no association between left-right preference and the animal (zebra, panda) assigned to be good $\left(p_{\text {rep }}=0.76\right)$.

In the vertical task, by contrast, a majority of both left-handers (89\%) and right-handers (83\%) drew the good animal in the top box and the bad animal in the bottom box (left-handers: sign test on 8 top vs. 1 bottom, $p_{\text {rep }}=0.93$; right-handers: sign test on 88 top vs. 18 bottom, $p_{\text {rep }}=0.99$; see Figure 1d). By Fisher's exact test and binary logistic regression, there was no reliable association between the handedness of the participant and the top-bottom placement of the good and bad animals ( $p_{\text {rep }}$ for the exact association $=0.74 ; \mathrm{OR}=0.61,95 \% \mathrm{CI}=0.07-5.19)$.

In short, the association between horizontal space and valence is body-specific: The number of participants who placed the good animal in the box on their dominant side outnumbered those who placed it on their nondominant side by a ratio of more than 2 to 1 . Right-handers' responses were consistent with the mental metaphor Good Is Right, and left-handers' with the mental metaphor Good Is Left. The association between vertical space and valence is different. People reliably apply the mental metaphor Good Is Up even when the task does not require them to activate any linguistic metaphors for valence or to use language at all in their response. This is true regardless of handedness. Thus, the effect of handedness in the horizontal task cannot be due to differences between right- and left-handers' space-valence mappings, in general; rather, it is specific to the space-valence mappings that were predicted to vary on the basis of the body-specificity hypothesis.

\section{Experiment 2: Do People Know That Handedness Influences Their Judgments?}

In Experiment 1, more than two thirds of participants placed the good animal in the box on their dominant side. Were they aware that their responses corresponded to their handedness? Data collection procedures did not allow for an explicit debriefing, so it is possible that participants associated the good and bad animals with their dominant and nondominant sides strategically. To find out whether people were conscious of the handedness manipulation, I replicated the horizontal diagram task in a new group of participants but included a written debriefing and two additional changes. First, for the debriefing, two of the filler questions that followed the diagram task were replaced with free-response questions asking participants to guess the purpose of the task and to explain their answer. Second, an objective measure of handedness was included to corroborate participants' self-report. Finally, the task was administered to native speakers of Dutch in the Netherlands, to generalize the findings of Experiment 1 to a new population. As in English, conventions in the Dutch language and culture associate good with right but not with left.

\section{Method}

Participants. Students from the Radboud University and University of Amsterdam communities participated $(N=100 ; 14$ left-handers, 86 right-handers by self-report and by observed writing/drawing hand).

Materials and procedure. The horizontal diagram questionnaire from Experiment 1 was used. Instructions were translated into Dutch by a native-Dutch-speaking linguist who is fluent in English. After completing the questionnaire, participants gave written responses (in Dutch) to three questions: one filler ("What is your major in school?"), and two free-response debriefing questions. First, they answered "What do you think this experiment was about? What do you think it was testing?" and then "Why do you think you placed the good animal in the box that you did?" There was no mention of handedness among the printed questions.

Handedness was assessed in two ways. Participants were tested individually or in small groups. Unbeknownst to the participants, the experimenter observed and recorded the hand they used to draw the animals and write their responses. After the participants had completed the diagram task and the written posttest questions, the experimenter asked them to write the word right or left at the bottom of the page to indicate whether they were right- or lefthanded.

\footnotetext{
${ }^{2} p_{\text {rep }}$ indicates the probability of replicating an observed effect, given an equipotent replication (Killeen, 2005). A $p_{\text {rep }}$ value of .92 corresponds to a $p$ value of .05 and can be interpreted as estimating a $92 \%$ probability of a replication producing a difference with the sign in the same direction as the observed difference.
} 


\section{Results and Discussion}

As in the previous experiment, a majority (71\%) of left-handers drew the good animal in the box on the left of the cartoon character ( ign test on 4 right side vs. 10 left side, $p_{\text {rep }}=0.83$ ), whereas a majority $(66 \%)$ of right-handers drew the good animal in the box on the right ( $\operatorname{sign}$ test on 57 right side vs. 29 left side, $p_{\text {rep }}=0.99$; see Figure 2a). There was a significant correlation between the handedness of the participant and the left-right placement of the good and bad animals by Fisher's exact test ( $p_{\text {rep }}$ for the exact association $=0.99$ ) and binary logistic regression of left-right preference on handedness $(\mathrm{OR}=4.91 ; 95 \% \mathrm{CI}=1.42-17.03)$, indicating that right-handers were nearly five times more likely than left-handers to place the good animal on the right and the bad animal on the left.

Due to counterbalancing, approximately equal numbers of participants were instructed to draw the good animal first (43 righthanders, 9 left-handers) and the bad animal first (43 right-handers, 5 left-handers). When the good animal came first, $69 \%$ of participants placed the good animal on their dominant side. Likewise, when the bad animal came first, $65 \%$ of participants placed the good animal on their dominant side. The proportions of bodyspecific responses did not differ significantly as a function of the temporal order in which participants drew the good and bad animals $\left(p_{\text {rep }}=0.75\right)$. There was also no association between left-right preference and the animal (zebra, panda) assigned to be $\operatorname{good}\left(p_{\text {rep }}=0.79\right)$.

For 100 out of 100 participants, the hand that the experimenter recorded as the participant's writing/drawing hand matched their

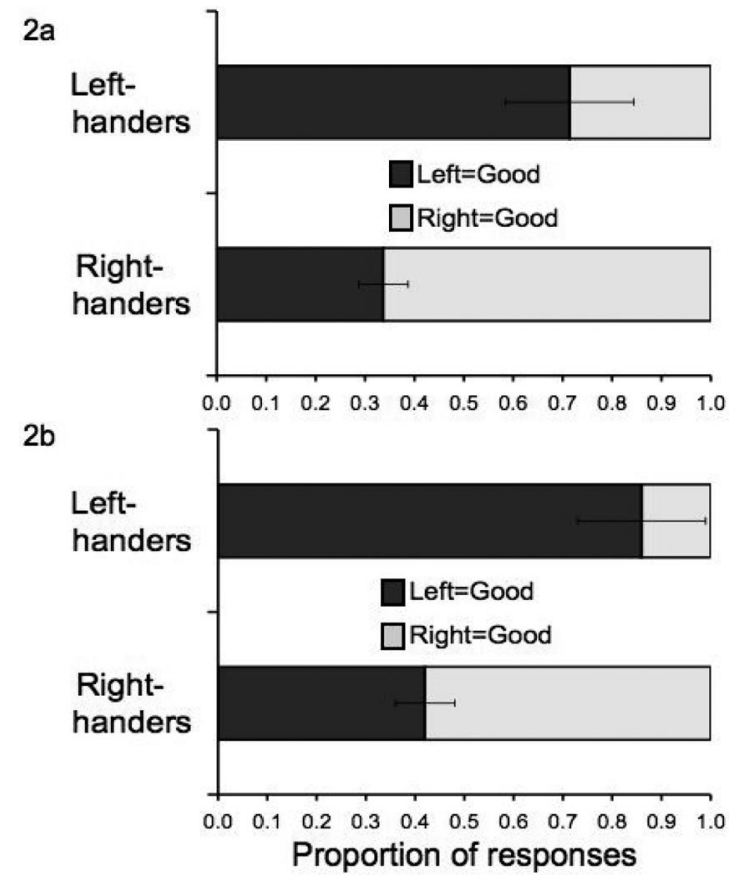

Figure 2. Results of Experiments 2 and 3. 2a: Proportion of left- and right-handers in Experiment 2 who drew the good animal in the left box (dark bars) and the right box (light bars). 2b: Proportion of left- and right-handers in Experiment 3 who indicated that the good animal should be placed in the left box (dark bars) and the right box (light bars). Error bars indicate standard error of the mean. self-reported handedness. Surreptitiously observing participants' handwriting is an efficient, objective, and reliable means of establishing handedness, given that writing hand accounts for the majority of the variance in the results of more extended measures such as the Edinburgh Handedness Inventory (Oldfield, 1971; S. M. Williams, 1986). The present result corroborates previous research validating simple binary self-report as a measure of handedness and showing strong agreement between single-item self-report and other measures (Coren, 1993).

Only 1 person out of the 100 participants guessed that the purpose of the experiment had anything to do with handedness. The most common response to the first debriefing question was to interpret the task at face value as investigating whether good and bad are more strongly associated with one side or the other $(n=$ $31)$. The second most common response was "no idea" $(n=21)$, followed by several speculations about hemispheric dominance for memory or emotions, effects of writing direction, political preference, or preferences for one animal or the other. In total, participants offered 29 distinct explanations of the task that had nothing to do with handedness.

When asked to speculate further about why they placed the good animal in the box that they did, 8 participants offered handedness as the explanation, and 6 more mentioned handedness among other alternative explanations or after indicating that they were not certain about this conjecture-or that they were guessing wildly. Among these 14 participants (14\% of the total sample), there were equal proportions of right-handers $(12 / 86 ; 14 \%)$ and left-handers $(2 / 14 ; 14 \%)$. The fact that 13 of the 14 did not mention handedness when asked to explain what the experiment was testing suggests that handedness came to mind later, when they were asked to rationalize their response. It seems unlikely that they were thinking about handedness when making their responses in the diagram task. Importantly, the body-specific association between handedness and left-right preference remained when these 14 participants were removed from the analysis ( $p_{\text {rep }}$ for the exact association $=$ $0.92 ; \mathrm{OR}=3.10,95 \% \mathrm{CI}=0.86-11.25)$.

In summary, the results of Experiment 2 provide nearly an exact replication of Experiment 1 in a new population of participants. The objective handedness measure validates the self-report measure used in Experiment 1 (and in subsequent experiments reported here). The debriefing indicated that $99 \%$ of the participants were unaware of the purpose of the experiment, suggesting that the observed pattern of body-specific associations between space and valence does not depend on conscious or strategic awareness of handedness.

\section{Experiment 3: Does Handedness Affect Judgments When} People Don't Use Their Hands?

Why did right- and left-handers associate good with their dominant side in Experiments 1 and 2? On a skeptical interpretation, these results could reflect a task-related preference for the side of space that is easiest to interact with in the moment, while writing and drawing with one's dominant hand. Experiment 3 investigated whether a similar pattern of body-specific preferences is found when the participants respond without using their hands. 


\section{Method}

Participants. Students from the Radboud University community participated $(N=87 ; 7$ left-handers, 80 right-handers by self-report).

Materials and procedure. Participants performed a version of the horizontal diagram task used in Experiments 1 and 2. Rather than responding by drawing animals in boxes, they responded orally to indicate where they would place the good and bad animals. The picture of the cartoon character and empty boxes used in the previous tasks was printed in the center of a page. At the top of the page sentences were printed in Dutch, indicating which animal the character liked and which he disliked (order of mention and assignment of good and bad animals were counterbalanced across participants). A native-Dutch-speaking experimenter approached participants one at a time and explained the task while showing them the diagram, which was on one side of a notebook cover. At the moment that the experimenter was asking the participant to respond, she folded the notebook cover, hiding the diagram-ostensibly so that she could record the responses on the data sheet. This forced the participant to indicate which boxes the good and bad animals should be placed in orally, rather than by pointing, since the diagram was no longer visible. If the response was not clear, the experimenter asked for clarification until the participant used the Dutch words for "right" or "left" to designate at least one of the boxes. After completing the diagram task, the experimenter asked participants to report whether they were right- or left-handed, among three filler questions. All participants were tested in one extended data collection session, in one location on one day, to minimize the chance that participants tested later would have heard anything about the experiment from participants tested earlier.

\section{Results and Discussion}

Consistent with the results of the previous experiments, a majority $(86 \%)$ of left-handers indicated that the good animal should go in the box to the left of the cartoon character (sign test on 1 right side vs. 6 left side; Fisher's exact $p_{\text {rep }}=0.83$ ), whereas a majority $(58 \%)$ of right-handers indicated that it should go in the box on the right ( ign test on 46 right side vs. 34 left side, $p_{\text {rep }}=0.81$; see Figure $2 \mathrm{~b}$ ). There was a significant association between the handedness of the participant and the left-right placement of the good and bad animals ( $p_{\text {rep }}$ for the exact association $=0.94$; $\mathrm{OR}=8.11$; $95 \% \mathrm{CI}=0.93-70.53)$.

Due to counterbalancing, approximately equal numbers of participants were instructed to place the good animal first (42 righthanders, 3 left-handers) and the bad animal first (38 right-handers, 4 left-handers). The proportions of body-specific responses did not differ significantly as a function of the temporal order in which participants responded about the good and bad animals ( $p_{\text {rep }}=$ $0.75)$, or as a function of which animal was assigned to be good $\left(p_{\text {rep }}=0.77\right)$.

The pattern of body-specific responses was compared across Experiments 2 and 3. Overall, $67 \%$ of participants placed the good animal on their dominant side in Experiment 2, compared with $60 \%$ in Experiment 3 ( $p_{\text {rep }}=0.90$, corresponding to a $p$ value of $.07)$. According to a traditional significance threshold of $p<.05$, this result shows a marginally significant difference in the strength of body-specific space-valence associations across experiments. This could indicate that using one's dominant hand during the task increases the strength of the effect. However, this comparison must be interpreted cautiously, in part because of the small number of left-handers in the Experiment 3 sample. Moreover, whereas righthanders showed a stronger body-specificity effect in the task where they used their dominant hand to respond, left-handers showed a stronger effect in the task in which they responded orally (albeit neither of these differences was significant). Further experiments are needed to determine conclusively whether using one's hands in the moment affects left-right valence judgments.

What is clear from Experiment 3 is that handedness influences people's judgments even when they're not using their hands to perform the task. This suggests that the results of Experiments 1 and 2 were not an artifact of the response modality and cannot be explained in terms of performance factors related to which side of the page is more convenient to interact with in the moment. Rather, they reflect long-term associations between goodness and one's dominant side of space. Attesting to the robustness of these associations, the body-specific Good Is Left mapping was activated in left-handers even when the task required them to use words for "right" and "left" in the response, which could have activated the Good Is Right mapping found in language and culture, instead.

\section{Experiment 4: Body-Specific Judgments of Aliens' Attributes}

Do mental metaphors from space to valence influence how we evaluate things we encounter in different spatial locations? In Experiments 1-3, right- and left-handers demonstrated bodyspecific space-valence mappings when they were required to assign good and bad stimuli to spatial locations, explicitly. Experiment 4 tested for implicit influences of the left-right position of stimuli on right- and left-handers' judgments of positive or negative traits in alien creatures.

\section{Method}

Participants. Students from the Stanford University and the University of California, Riverside, communities participated in exchange for payment or course credit $(N=286$; 40 left-handers, 246 right-handers by self-report).

Materials and procedure. Participants were given a one-page paper-and-pencil questionnaire in a packet of unrelated questionnaires. Instructions welcomed participants to the planet Fribbalia and asked them to make judgments about some of its inhabitants. Below the instructions were 12 pairs of Fribbles (alien creatures created by Michael J. Tarr, Brown University, www.tarrlab.org). Members of each Fribble pair were visually similar but clearly distinguishable by several salient features. Fribbles were arranged in two columns, one on each side of a list of questions printed in a center column. This arrangement placed the members of each Fribble pair on opposite sides of the page without calling attention to their spatial arrangement.

Participants were asked to circle one member of each Fribble pair, located on either the left or right side of the question, to indicate a judgment about one of four personal characteristics (i.e., intelligence, attractiveness, honesty, happiness), for a total of 12 judgments per participant. Participants were randomly given either 
the positive-wording version of the questionnaire (i.e., "Circle the Fribble who looks more intelligent"/"more attractive"/"more honest"/"happier") or the negative-wording version (i.e., "Circle the Fribble who looks less intelligent"/"less attractive"/"less honest"/ "sadder"). Both positive- and negative-polarity questions were used for two reasons. First, it is known that question polarity can influence scalar judgments in general (Clark, 1969), so using both positive and negative questions increases the generality of the results. Second, and more importantly, using both positive and negative wording allowed the valence of the response to be orthogonal to its location: For example, it was possible to make a happiness judgment consistent with the Good Is Right mapping either by circling the right Fribble in the positive wording condition (indicating that the right Fribble was happier) or by circling the left Fribble in the negative wording condition (also indicating that the right Fribble was happier). As such, it was possible to determine whether observed effects of stimulus location were due to participants' evaluation of the stimuli per se or simply to their preference to make circles on one side of the page versus the other, regardless of their assessment of the Fribbles' relative virtues.

The assignment of questions to Fribble pairs was counterbalanced across participants, and crucially, assignment of the members of each pair to the right or left side of the page was also counterbalanced to ensure that interitem differences could not result in spurious preferences for Fribbles located on the left or right side of the page (even if one Fribble of a pair was inherently more appealing than the other). After completing the Fribble task, participants were asked to report their handedness, among other demographic and filler questions. The questionnaire took approximately $1 \mathrm{~min}$ to complete.

\section{Results and Discussion}

For each of the 286 participants, the number of responses attributing positive characteristics to Fribbles on the right versus the left side of the page was tabulated. A total of 210 participants (74\%; 31 left-handers, 179 right-handers) showed either a rightward or a leftward preference on average, and 76 participants (26\%; 9 left-handers, 67 right-handers) showed no preference, selecting six Fribbles from the left column and six from the right column. The proportion of participants who showed no preference did not differ as a function of handedness (27\% of left-handers, $23 \%$ of right-handers), $\chi^{2}(1)=0.24$, Fisher's exact $p_{\text {rep }}=0.63$, so these participants' data were excluded from further analysis.

Of the participants who showed a directional preference, a majority of left-handers $(65 \%)$ attributed positive characteristics more often to Fribbles on the left of the page, whereas a small majority of right-handers $(54 \%)$ attributed positive characteristics more often to Fribbles on the right of the page (left-handers: sign test on 11 right-side preference vs. 20 left-side preference, $p_{\text {rep }}=0.85$; right-handers: sign test on 96 right-side preference vs. 83 left-side preference, $p_{\text {rep }}=0.74$ ). As in the previous experiments, there was a reliable association between participants' handedness and their preference for Fribbles on the left versus the right of the page ( $p_{\text {rep }}=0.94$; see Figure 3a). Binary logistic regression of leftright preference on handedness showed that the right-handers were about twice as likely as the left-handers to attribute more positive characteristics to Fribbles on the right side of the page (OR estimate $=2.10 ; 95 \% \mathrm{CI}=0.95-4.64)$.

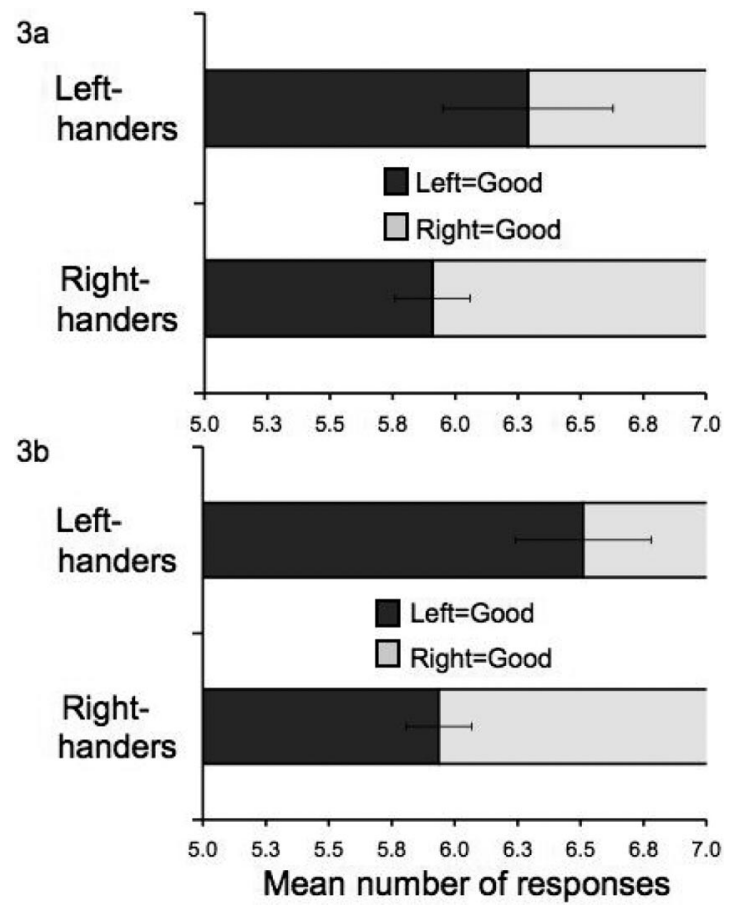

Figure 3. Results of Experiments 4 and 5. 3a: Mean number of responses (out of 12) made by left- and right-handers in Experiment 4 (Fribbles task) indicating a preference for the Fribble on the left (dark bars) and the Fribble on the right (light bars). 3b: Mean number of responses (out of 12) made by left- and right-handers in Experiment 5 (shopping and jobs tasks) indicating a preference for the description on the left (dark bars) and the description on the right (light bars). Error bars indicate standard error of the mean.

Was this body-specific effect simply due to left-handers preferring to write responses on the left of the page and right-handers on the right? The positive and negative wordings required participants to make circles on different sides of the page to indicate which Fribbles had more positive characteristics. In a chi-square analysis, the pattern of body-specific responses was compared across the positive-wording and negative-wording versions of the questionnaire, in right- and left-handers combined. There was no reliable association between the wording of the questionnaires and the frequency with which participants' responses followed the Dominant Side Is Good mental metaphor, $\chi^{2}(1)=0.44, p_{\text {rep }}=0.68$. Thus, the two versions of the questionnaire showed similar results. This rules out the possibility that participants simply circled Fribbles on the side of the page that was most convenient for them to reach. Rather, in judging intelligence, attractiveness, honesty, and happiness, participants were influenced by the left versus right location of the Fribbles, per se.

In summary, the right-left location of Fribbles influenced participants judgments of their personal characteristics differently in right- and left-handers, even though spatial location was irrelevant to the judgments. Among the majority of participants who showed a directional preference, left-handers were more likely than righthanders to show a Good Is Left bias, and right-handers were more likely than left-handers to show a Good Is Right bias. These results support the body-specificity hypothesis. 


\section{Experiment 5: Body-Specific Decisions About the Office and Marketplace}

Experiment 5 was designed to determine whether the bodyspecific influences of spatial location apply not only to alien creatures but also to the kinds of everyday decisions that we make here on Earth. Rather than making decisions on novel pictures with no preexperimental semantic content, participants judged brief verbal descriptions of job applicants (in the job task) and familiar products that might be advertised in a newspaper or website (in the shopping task).

\section{Method}

Participants. Students from the Stanford University and the University of California, Riverside, communities participated in exchange for payment or course credit $(N=371 ; 50$ left-handers, 321 right-handers by self-report).

Materials and procedure. The participants were given a paperand-pencil questionnaire on two sides of a page, among unrelated questionnaires. On one side of the page was the job task. Participants were asked to imagine that they were in charge of hiring new personnel for their company and that they had narrowed the choices to two candidates for each of six jobs (programmer, graphic designer, security guard, advertising copywriter, administrative assistant, and child daycare manager). Their job was to decide whom to hire on the basis of brief descriptions of the candidates arranged in columns on the left and right sides of the page (as in the Fribbles task). The names of the jobs were listed in the center of the page, separating the pairs of descriptions to the right and left without calling attention to their spatial location. For each job, participants were instructed to circle the description of the candidate they would be more likely to hire and to cross out (with an $X$ ) the description of the candidate they would be less likely to hire. Descriptions were intended to make candidates comparable yet distinctive. For example, one applicant for the programmer position was an engineering major from Virginia Tech who programs in Perl, and the other was a math major from Georgia Tech who programs in Python.

On the other side of the questionnaire was the shopping task, identical in design to the job task. Participants were asked to imagine that they were shopping for six products (dish soap, mattress, carpet, desk chair, family car, inflatable kiddie pool) and that they had searched the Internet and narrowed their choices to two competing brands for each product. Now their task was to decide which product to buy on the basis of brief product descriptions, which were listed in columns on the right and left of the product names. For example, one brand of dish soap boasted that it killed germs, was fresh-scented, and moisturized hands, and the other that it was antibacterial, was clean smelling, and conditioned skin. For each item, participants were instructed to circle the description of the product they would be more likely to buy and to cross out (with an $X$ ) the description of the product they would be less likely to buy.

For both the job and shopping tasks, participants made an equal number of responses on the right and left of the page, one circle and one cross for each pair of descriptions. The order in which they were instructed to make circles and crosses was counterbalanced across subjects, so preference (preferred item, dispreferred item) was orthogonal to the temporal primacy of responses (first item circled or crossed out, second item circled or crossed out). The order of the job and shopping tasks was also counterbalanced across subjects, as was the assignment of the members of each pair of descriptions to the right or left side of the page, to ensure that interitem differences could not result in spurious preferences for items located on the left or right side of the page. Each participant made a total of 12 responses. After completing both tasks, participants were asked to report their handedness, among other demographic and filler questions. The questionnaire took 1-2 $\mathrm{min}$ to complete.

\section{Results and Discussion}

Responses to the job and shopping questions were combined for all analyses. For each of the 371 participants, the number of people and products selected on the right side versus the left side of the page was tabulated. A total of 272 participants $(73 \%$; 35 left-handers, 237 right-handers) showed either a rightward or a leftward preference on average, and 99 participants (27\%; 15 left-handers, 84 right-handers) showed no preference, selecting six items from the left column and six from the right column. The proportion of participants who showed no preference did not differ as a function of handedness (30\% of left-handers, $26 \%$ of right-handers), $\chi^{2}(1)=0.18$, Fisher's exact $p_{\text {rep }}=0.62$, so these participants' data were excluded from further analysis.

Of the participants who showed a directional preference, a majority of left-handers $(74 \%)$ attributed positive characteristics more often to people or products on the left side of the page, whereas a small majority of right-handers (52\%) attributed positive characteristics more often to items on the right side of the page (left-handers: sign test on 10 right-side preference vs. 29 left-side preference, $p_{\text {rep }}=0.99$; right-handers: sign test on 123 right-side preference vs. 114 left-side preference, $p_{\text {rep }}=0.64$ ). There was a reliable association between handedness and preference for descriptions on the left versus the right of the page by Fisher's exact test ( $p_{\text {rep }}$ for the exact association $=0.99$; see Figure $3 b$ ). Binary logistic regression showed that right-handers were more than twice as likely as left-handers to attribute more positive characteristics to people or products described on the right side of the page (OR estimate $=2.69 ; 95 \% \mathrm{CI}=1.25-5.76$ ). These results demonstrate implicit body-specific preferences, corroborating the results of Experiment 4.

A final set of analyses was conducted with the pooled data from Experiments 1-5 to assess the association of handedness and left-right preference overall. When all participants in the horizontal condition of Experiment 1, all in Experiments 2-3, and all who showed a left-right preference in Experiments 4-5 were combined ( $n=777 ; 110$ left-handers, 667 right-handers), the results showed that $59 \%$ of participants made judgments consistent with the predictions of the body-specificity hypothesis (sign test on 458 with the hypothesis vs. 319 against the hypothesis, $p_{\text {rep }}=0.99$ ). Of the left-handers, $72 \%$ showed a Good Is Left preference (sign test on 31 right side vs. 79 left side, $p_{\text {rep }}=0.99$ ), and of the righthanders, 57\% showed a Good Is Right preference (sign test on 379 right side vs. 288 left side, $p_{\text {rep }}=0.99$ ). By Fisher's exact test, there was a highly significant association between handedness and left-right preference $\left(p_{\text {rep }}\right.$ for the exact association $=0.99$ ). Binary logistic regression showed that, overall, right-handers were 
more than three times as likely as left-handers to associate positive responses with the right side of the page (OR estimate $=3.35$; $95 \% \mathrm{CI}=2.15-5.21)$.

\section{General Discussion}

Five experiments demonstrated associations between horizontal space and the mental representation of abstract concepts with positive and negative emotional valence. These associations differed between right- and left-handers. Right-handers were more likely than left-handers to associate right with positive ideas and left with negative ideas. Left-handers were more likely than right-handers to associate left with positive ideas and right with negative ideas. Right- and left-handers tended to link good things like intelligence, attractiveness, honesty, and happiness with opposite sides of leftright space, each group associating them more strongly with their dominant side. By contrast, both left- and right-handers showed the same preference to associate good things with up and bad things with down. This pattern of results was predicted on the basis of the body-specificity hypothesis and demonstrates that people with different bodies (in this case, right- and left-handers) form correspondingly different mental representations, even in highly abstract conceptual domains.

\section{Distinguishing Influences of Language, Culture, and Body}

Previous experiments have been unable to determine whether mental metaphors from space to valence arise in the individual learner due to correlations in bodily experience or to correlations in linguistic experience. The vertical condition of Experiment 1 illustrates this difficulty. The data from this nonlinguistic task validate the mental metaphor Good Is Up, which is evident in many linguistic metaphors. Yet, like previous results that have revealed implicit mappings from vertical space to valence (e.g., Casasanto \& Dijkstra, 2009; Casasanto \& Nabieva, 2007; Meier \& Robinson, 2004; Stepper \& Strack, 1993), these data are equivocal regarding the origins of this mental metaphor. On an embodied interpretation, participants put the good animal on top because they have generalized associative links between bodily action and emotion (e.g., standing tall when we feel proud). But on an alternative interpretation, they put the good animal on top because they habitually use linguistic expressions that conflate space and valence (e.g., high on life), and this linguistic experience has led to the creation of links between nodes representing height and happiness in an amodal conceptual network. Because embodied theories and amodal alternatives make the same behavioral predictions (in this and many other cases), it is not clear to what extent the mental metaphor Good Is Up arises in individuals (a) from correlations between their perceptuomotor and emotional experiences or (b) from correlations between vertical space and valence in linguistic and cultural conventions. Everyone is exposed to both kinds of correlations during development, so it is likely that both direct bodily experience and linguistic/cultural experience contribute to the formation of Good Is Up. But the relative contributions of these sources of experience remain unknown.

The body-specificity hypothesis allows us to distinguish the influences of bodily and linguistic/cultural experience unequivocally for mappings from left-right space to valence. The contrasting mental metaphors exhibited by left- and right-handers in Ex- periments $1-5$ cannot be explained by conventions in language or culture. In English-speaking cultures (and many others), cultural and linguistic conventions associate the right with ideas and actions that are good or allowable and the left with ideas and actions that are bad or prohibited. Conversely, there appear to be no linguistic or cultural conventions that link the left with things that are good. ${ }^{3}$ Both enculturation and body-specificity could potentially account for the Good Is Right mapping, which was stronger in right-handers, but only body-specificity can explain (a) the Good Is Left mapping found in the majority of left-handers and (b) the difference between right- and left-handers' judgments.

\section{Experience and Mental Simulation of Experience}

The question of whether concepts and word meanings are embodied must be addressed on at least two time scales: How did they develop in the individual learner, and how are they instantiated online during thinking and language use? An answer to the former question concerning concepts' experiential origins seems a prerequisite for the latter concerning the representational format in which experiences are recapitulated online. In order to support the claim that instantiating concepts requires people to create modalityspecific mental simulations of physical actions or bodily states (see Barsalou, 1999; Feldman, 2006; Gallese \& Lakoff, 2005; Goldstone \& Barsalou, 1998; Prinz, 2002) one must specify the actions or states that could serve as their basis.

The present data provide evidence that abstract concepts with positive and negative valence are embodied insomuch as their mental representation depends, in part, on particulars of an individual's body: possibly on the mere fact of having the bodily trait of being right- or left-handed but presumably also on correlations between emotional states and lateralized physical actions. Thus, at least some abstract concepts have an embodied origin. It remains to be determined whether these concepts also have an embodied instantiation online, in modality-specific regions of the brain.

\section{Formation of Body-Specific Mental Metaphors}

What is the mechanism by which bodily differences cause body-specific mappings to emerge? It is proposed that the Good Is Right and Good Is Left mental metaphors are created in right- and left-handers, respectively, via correlational learning (Hebb, 1949) over a lifetime of lopsided perceptuomotor experience. People come to implicitly associate good things more strongly with the side of space they can interact with more fluently (their dominant side) and bad things with the side of space they interact with less fluently (their nondominant side).

This proposal is consonant with other experimental data linking perceptuomotor fluency with positive affective judgments (Beilock \& Holt, 2007; Oppenheimer, 2008; Reber et al., 1998; Van den Bergh et al., 1990), and it makes a number of testable predictions. For example, if asymmetries in perceptuomotor flu-

\footnotetext{
${ }^{3}$ One possible exception to the general rule that "left is bad" in linguistic and cultural conventions is that in the United States and some European countries, liberal political views are said to be on the left of the political spectrum. However, the valence of this spatial-political mapping is not fixed with respect to positive or negative valence; rather, whether "politically left" is good or bad varies according to the politics of the individual.
} 
ency give rise to body-specific associations between space and valence, then preferences for the dominant side of space should be strongest in individuals who are strongly "handed" and weakest in individuals who are ambidextrous (Oldfield, 1971). Furthermore, the development of body-specific mental metaphors in children should be linked to the emergence of their handedness. Finally, if body-specific mental metaphors from space to valence result from asymmetries in perceptuomotor experience (and assuming that the resulting associations remain somewhat plastic throughout the lifetime), then changes in the body that necessitate systematic changes in the way an individual interacts with the physical environment should produce corresponding changes in the strength of implicit directional preferences: A right-hander whose dominant hand is injured or lost should, over time, exhibit a weakening or even a reversal of the Good Is Right mapping. Further experiments are needed to test these predictions.

An alternative possibility is that body-specific mental metaphors have a purely genetic origin, and their development is not mediated by asymmetric perceptuomotor activity. Rather, that which gives rise to handedness also gives rise to contrasting directional preferences in left- and right-handers (see the Are the Neural Substrates of Affect and Motivation Body-Specific? section below). The proposal that body-specific space-valence mappings are based entirely on genes rather than physical experience seems difficult to motivate, but it is not ruled out by the present data, which can only establish a correlation between handedness and preference. Importantly, the data presented here would still support the main theoretical claim of body-specificity (that people with different bodies think differently in corresponding ways) and the main empirical claim of this article (that right- and left-handers have body-specific mental metaphors for valence), even if body-specific preferences were found to be activity-independent.

\section{Relationships Between Mental Metaphors and Linguistic Metaphors}

Why is the right side conventionally the good side across many languages and cultures? The fact that left- and right-handers adhere to the same linguistic and cultural conventions may appear, at first, to argue against the possibility that these conventions have a bodily origin. In principle, linguistic and cultural conventions could always reflect the body-specific mental metaphors of their users, but this is not the case: Left-handers do not greet others with left-handed handshakes or refer to a correct response as "the left answer." Consider the confusion that could ensue if this were the case: When someone told you that your answer was "right," you would need to find out whether the speaker was right- or lefthanded in order to determine whether your answer was correct or incorrect! A tacit agreement among members of a community to conform to linguistic and cultural conventions that reflect the implicit body-specific mental metaphors of the majority avoids confusion and facilitates communication. Right-handers predominate in the population worldwide. Thus, the finding of a bodyspecific preference for the right in right-handers provides a potential explanation for the prevalence of the Good Is Right mapping across languages and cultures.

Good Is Left is a mental metaphor without any corresponding linguistic metaphors. This discovery, coupled with the ubiquity of the Good Is Right mapping, has implications for language-based approaches to conceptual structure (e.g., Evans, 2004; Lakoff \& Johnson, 1980, 1999; Talmy, 1988). Linguistic data could be interpreted as evidence that (a) people conceptualize goodness metaphorically in terms of rightward space, (b) the Good Is Right mental metaphor is universal, both within and across cultures, and (c) Good Is Left does not exist as a mental metaphor; its existence is made doubtful by the absence of supporting linguistic metaphors and is ruled out by the presence of conflicting "left is bad" idioms across language and cultures. But the present data challenge all three of these conclusions. We must be cautious when trying to infer conceptual structure from patterns in language and to discover conceptual universals on the basis of linguistic universals.

It is possible, in principle, that people have two mental metaphors from right-left space to valence, one based on patterns in language and culture and the other on patterns of direct bodily experience. If so, the two would be congruent for right-handers but incongruent for left-handers (for whom language and culture associate good with right, but bodily experience associates good with left). This makes a simple prediction: Assuming the influences of the two metaphors on an individual's behavior are roughly additive, the Good Is Right bias in right-handers should be stronger than the Good Is Left bias in left-handers. But this prediction is not supported by the results of Experiments 1-5. On the contrary, across experiments, the body-specific pattern tended to be stronger in left-handers than in right-handers (see the How Does "Good Is Left" in Lefties Compare With "Good Is Right" in Righties? section below). These data do not provide any evidence for an implicit Good Is Right mental metaphor based on patterns in language and culture that are shared by both right- and lefthanders.

Does the existence of linguistic expressions like "the right answer" imply the existence of the mental metaphor Good Is Right in all English speakers (including left-handers), despite the absence of evidence in the present data? Not necessarily. Expressions linking right with "good" do not appear to be metaphors but rather polysemies or frozen idioms (Keysar \& Bly, 1995; Murphy, 1996, 1997). The fact that speakers understand "the right answer" does not entail that they do so by activating associative mappings from the source domain of horizontal space; right in this sense may be understood nonmetaphorically. As such, left-handers should understand these linguistic expressions as easily as do right-handers, despite the difference in their implicit mental metaphors.

Productivity and systematicity are hallmarks of the kinds of linguistic expressions that are understood via mental metaphors (Lakoff \& Johnson, 1980, 1999). These traits are evident in linguistic metaphors that associate vertical space and valence. The idea "he got happier" can be expressed with a conventional metaphor like "his spirits rose" but also with a variety of related metaphors that are likely to be understood effortlessly even if they are novel (e.g., "his spirits soared," "sailed," "climbed," "skyrocketed"). Furthermore, these expressions linking up with "happy" are systematically related to expressions linking down with "unhappy" (e.g., "his spirits sank," "plunged," "plummeted," "nosedived"). Degrees of happiness or sadness can be expressed as locations along a vertically spatialized happiness continuum.

By contrast, linguistic expressions that associate horizontal space and valence tend to be unproductive and unsystematic. The idiom "I've got two left feet" means "I'm clumsy," but this meaning is lost if the expression is varied even slightly (e.g., "I've 
got two left soles," "two left ankles," "two left toes," "two left shoes"). Furthermore, having two left feet does not indicate a position along a horizontally spatialized clumsiness continuum. "I've got two right feet" does not mean "I'm graceful." Likewise, "the left answer" does not mean "the wrong answer." "My righthand man" means "a close associate," but "my left-hand man" does not mean "a distant associate"; "out in left field" means "eccentric," but "out in right field" does not mean "mainstream." If space is used unsystematically as in these right-left idioms, then spatial relations cannot be used to support inferences about relative goodness or badness. As such, it is unlikely that these linguistic expressions are understood metaphorically via mappings from space to valence.

Right-left idioms in language and culture that express ideas with positive and negative valence may be related to body-specific mental metaphors in right-handers. But based on the nature of these idioms in English and Dutch, and on the experimental data presented here, it appears that this relationship is largely historical. This is true, at least, for English speakers in California and Dutch speakers in the Netherlands. In cultures with strong left-hand taboos, where left-right valence mappings are more salient, these cultural conventions could give rise to active mental metaphors (Kövecses, 2005). If so, it may be possible to observe additive relationships between culture-specific and body-specific mappings. For example, left-handers in Islamic cultures might show an attenuation of the body-specific preference for the left (which conflicts with the strong culture-specific Bad Is Left metaphor), and right-handers an accentuation of the body-specific preference for the right (which is supported by the culturally constructed Good Is Right metaphor).

\section{How Does "Good Is Left" in Lefties Compare With "Good Is Right" in Righties?}

Across all five experiments, there was a tendency for the Good Is Left mapping in left-handers to be stronger than the Good Is Right mapping in right-handers. The strength of the body-specific preference differed significantly between groups only in Experiment 5 (Fisher's exact test for the difference between the proportions of body-specific responses in left- and right-handers, $p_{\text {rep }}=$ 0.99 ) and in the combined data from all participants who showed a left-right bias in Experiments 1-5 ( $p_{\text {rep }}=0.99$ ). This finding was unexpected and should be interpreted with caution for a combination of reasons, foremost of which is that this unpredicted trend was nonsignificant in four out of five experiments. Furthermore, the large difference in the number of left- and right-handed participants complicates comparison of the strength of bodyspecific preferences across groups.

Two potential explanations for the observed pattern merit further investigation. First, asymmetries in perceptuomotor experience may be more salient for left-handers, who are habitually inconvenienced by customs and devices designed for righthanders. More salient perceptuomotor asymmetries could result in stronger associations between actions with the dominant hand and experiences with positive emotional valence. Second, culturespecific mental metaphors may be interacting with the bodyspecific left-right spatialization of valence through a blending of three metaphorical mappings. According to the implicit mental number line in English speakers, the first in a series (or a pair) is the leftmost (Dehaene, Bossini, \& Giraux, 1993). Linguistic expressions like "the prime example" conflate primacy with goodness (i.e., this phrase can mean the first example, the best example, or both). Speakers of languages like English may be predisposed to consider the leftmost item to be the first and therefore the best. This metaphorical blend of left, first, and best should result in a culturally constructed Good Is Left bias in all participants, which would reinforce the body-specific Good Is Left bias in left-handers but work against the body-specific Good Is Right bias in right-handers.

\section{Body-Specific Interpretation of Previous Findings}

Studies from disparate literatures have reported associations of direction, preference, and handedness in specialized domains such as spatial navigation, music perception, and facial emotion processing. Body-specificity may provide a unifying account of these data. Patrons in American museums reportedly show a strong bias to turn right when they enter a gallery (Robinson, 1933). This is true in spite of museum curators' tendency to create exhibits that begin on the left and proceed rightward, consistent with the direction of reading and writing in English. Scharine and McBeath (2002) tested two explanations for the right-turn bias: handedness and driving experience. Right- and left-handed American participants (who drive on the right side of the road) and English participants (who drive on the left) performed a simple $\mathrm{T}$ maze. They were asked to walk down an aisle formed by two parallel sets of bookshelves in a university library and to find the target (a sticky note) hidden at the end of one of the bookshelves. There were significant effects of driving experience: The Americans were more likely to turn right at the end of the bookshelves, and the English to turn left. But the strongest predictor of turning preference was handedness: Both American and British participants were biased to search for the target by turning toward their dominant side. Scharine and McBeath characterized this finding as a "locomotive directional bias" (2002, p. 249), which may be limited to spatial navigation. Alternatively, this turning bias may be one instance of a more general body-specific preference for things located on one's dominant side of space.

Affective judgments about music also depend, in part, on location and handedness. McFarland and Kennison (1989) played right- and left-handers excerpts from instrumental musical pieces that evoke positive or negative emotions. Excerpts were presented through a single headphone placed over the participant's right or left ear. Right-handers reported experiencing more positive emotions when music was presented on the right; left-handers showed the opposite pattern, reporting more positive emotions when music was presented on the left.

Studies of emotional face processing show compatible results. In one experiment, right-handers tended to judge faces as expressing a more negative emotion when they appeared briefly on the left side, consistent with the Good Is Right mapping (Natale, Gur, \& Gur, 1983). In another experiment, left-handers rated faces as expressing a more positive emotion when they appeared on the left of the screen and a more negative emotion when they appeared on the right, consistent with the Good Is Left mappings (Everhart, Harrison, \& Crews, 1996).

It appears that no domain-general Good Is Left preference has been reported previously, but a Good Is Right preference consistent with the present results was discovered serendipitously by 
Wilson and Nisbett (1978). They asked participants to evaluate the quality of four nylon stockings that, unbeknownst to them, were identical in all but one respect: Each stocking had been treated with a different scent. The stockings were hung side-by-side on a rack with their left to right order counterbalanced across participants. Scent had no effect on participants' evaluations, but to the experimenters' surprise, the left to right position did: The farther an item was to the right of the row, the more likely it was to be selected as superior. Nisbett and Wilson did not know the cause of the rightward bias but speculated that it was a temporal order effect (since participants generally inspected the stockings serially from left to right). While temporal order may have been one influence, the present data suggest an alternative account: Perhaps participants preferred stockings on their dominant side. Given the predominance of right-handers in the population, most of Nisbett and Wilson's participants were probably right-handed, and so on average they would have preferred items on the right.

\section{Are the Neural Substrates of Affect and Motivation Body- Specific?}

The results of Experiments 1-5 suggest a possible reinterpretation of a well-established pattern in cognitive-affective neuroscience. Dozens of experiments have investigated the hemispheric laterality of emotional valence and of approach and avoidance behavior. Approach-related behavior (correlated with positive valence) has been lateralized to the left hemisphere, and avoidancerelated behavior (correlated with negative valence) to the right hemisphere, particularly in the frontal lobes (e.g., Ahern \& Schwartz, 1979; Cretenet \& Dru, 2004; Davidson, 1992; Davidson et al., 1990; Davidson \& Fox, 1982; Dimond, Farrington, \& Johnson, 1976; Killgore \& Yurgelun-Todd, 2007; Maxwell \& Davidson, 2007; Natale, Gur, \& Gur, 1983; Schiff \& Bassel, 1996). ${ }^{4}$ This pattern of hemispheric specialization has achieved the status of scientific fact. Yet, most of the studies that demonstrate the pattern have exclusively tested right-handed participants. Thus, the established laterality of positive/approach and negative/ avoidance behaviors may obtain only (or primarily) for righthanders.

On a body-specific interpretation of the available data, perhaps positive emotions and approach behaviors are not mediated by the left frontal lobe, universally; rather, perhaps they are mediated by the frontal lobe that controls the dominant hand. From early childhood, we tend to reach for (i.e., approach) things that we evaluate as positive and not to reach for things that we evaluate as negative. This gives rise to associations of positive stimuli and approach behaviors with the dominant hand (the hand we usually reach with), which could be mediated by the contralateral frontal lobe, in or near relevant motor areas.

Conversely, negative emotions and avoidance behaviors may be mediated by the frontal lobe that controls the nondominant hand. This requires a slightly different explanation, because it is unlikely that we prefer to reach for negative things with our nondominant hands (moreover, reaching is an approach behavior, no matter what we're reaching for). The association of our nondominant hand with negative stimuli and avoidance behavior may arise, in either evolutionary or developmental time, due to (a) the act of separating what we want from what we don't, (b) negative out- comes of actions with our nondominant hands, and (c) defensive actions.

First, if we use our nondominant hand to push away things that we don't want, this leaves the dominant hand free to grasp and manipulate things we that do. Imagine picking an apple or shelling a nut. To separate an apple from the tree, we're likely to hold the branch with our nondominant hand (pushing it away) while picking the fruit with our dominant hand (pulling it toward us). Likewise, after we crack a nut, we're likely to hold the shell (which we don't want) in our nondominant hand while picking out the meat with our dominant hand.

Second, even though we prefer to use our dominant hand for manipulating objects, sometimes we end up using our nondominant hand due to functional constraints: when the object is located on our nondominant side or when our dominant hand is busy. The outcome is often frustrating. Such experiences could discourage approach behaviors with our nondominant hands and help to establish an association between our nondominant side and negative outcomes.

Third, if we use our nondominant hand to fend off attack (an avoidance behavior), this leaves our dominant hand free to retaliate (an approach behavior) or to perform more complicated defensive actions. Sword fighters in previous centuries raised the shield with the nondominant hand while wielding the sword offensively with the dominant hand (Gould, 1908, in Scharine \& McBeath, 2002). Consistent with this proposal, Coren (1992) demonstrated a natural startle response in which right-handers raised their left hand higher than their right, and left-handers raised their right hand higher than their left, both groups shielding their faces with the nondominant hand in response to perceived danger.

If the hemispheric laterality of affective-motivational systems correlates with manual action tendencies, it should reverse between right- and left-handers. This proposal is speculative, but it is consistent with the music-processing and face-perception studies reviewed above. Due to the crossing of auditory and visual pathways, stimuli were processed initially by the hemisphere contralateral to the side on which they were presented. Thus, positive judgments could have resulted from initial processing by the right hemisphere in left-handers and by the left hemisphere in righthanders, consistent with a body-specific reversal of hemispheric specialization for positive and negative affect.

Although suggestive, these studies do not conclusively establish whether hemispheric specialization for positive and negative affective processing differs between right- and left-handers. Stimuli that were rated more positively were processed initially by the hemisphere that controls the dominant hand, but they were also presented on the participant's dominant side of space; thus, the side on which stimuli appeared was confounded with the hemisphere in which they were initially processed. As such, the finding that right- and left-handers produce contrasting affective judgments on laterally presented stimuli does not entail that affectivemotivational systems are lateralized differently in right- and left-

\footnotetext{
${ }^{4}$ Affective states and motivational states are correlated, but they are also separable. Maxwell and Davidson (2007) conducted a study exploring both positive/negative valence and approach/avoidance behaviors. In a footnote, they reported that the one left-handed participant they tested showed a reversal of the hemispheric specialization found in right-handers.
} 
handers' brains. The same data could be interpreted as evidence that right- and left-handers form different associations between left-right location and positive-negative emotion in long-term memory, despite similar neural localization for positive/approach and negative/avoidance behaviors.

The present experiments are also equivocal regarding the neural correlates of body-specific preferences for the left or right side of space. Presumably, the diagrams, Fribbles, and verbal descriptions were all processed bilaterally by the visual system. Written responses were programmed primarily by motor areas contralateral to the dominant hand, but it is unlikely that this difference between right- and left-handers was responsible for the observed effects, given that a similar pattern of results was found in Experiment 3 (where participants made no manual responses). We can conclude, on the basis of the present data, that selective processing of stimuli and responses by one hemisphere is not necessary to generate body-specific valence judgments. Beyond this, the neural underpinnings of body-specific preference judgments remain unclear.

If, indeed, the hemispheric laterality of affective-motivational systems were found to vary with hand dominance, this finding would contribute substantially to our understanding of relationships between emotion and action in the brain. A further question would concern the direction of causation between body-specific hemispheric specialization for affect and body-specific spacevalence associations. In principle, genetically determined hemispheric differences in affective processing between right- and left-handers could give rise to body-specific space-valence mappings. Alternatively, body-specific hemispheric specialization for affective processing could emerge over the course of development, on the basis of learned action-emotion correlations, as right-and left-handers use their dominant hand preferentially for positive/ approach behaviors and their nondominant hand for negative/ avoidance behaviors. Different action tendencies in right- and left-handers could be either an effect or a cause of differently lateralized neural systems for affect and motivation.

Further experiments in which neural activity can be observed more directly are needed to determine whether the hemispheric laterality of affective-motivational systems is a universal property of human brains - or nearly universal, like hemispheric specialization for language (Goodglass \& Quadfasel, 1954) — or whether affective-motivational systems are differently lateralized in rightand left-handers.

\section{Are Body-Specific Judgments Strategic?}

Demand characteristics are of potential concern in any task that requires explicit judgments. Wilson and Nisbett's (1978) stocking study described above was designed to investigate people's awareness of the factors that influence their preference judgments. They concluded that "subjects do not report [the influence of spatial position] or recognize it when it is pointed out to them" (1978, p. 124). Indeed, not one of their 52 participants mentioned the spatial position of stockings spontaneously when asked to explain their choice; however, they offered a total of 80 other spurious explanations. When asked directly about spatial position, all but one denied that it could have influenced their judgments.

Participants in Experiments 1-5, like those in Wilson and Nisbett's (1978) study, seem to have been unaware of the spatial and bodily factors influencing their judgments. Experiment 2 included a written debriefing that showed that 99 out of 100 participants were unaware that the purpose of the experiment had anything to do with handedness. Data collection procedures for the Fribbles, shopping, and job questionnaires did not allow participants to be debriefed in writing. But given that participants in the explicit spatial diagram task were largely unaware of the handedness manipulation (even though this task called attention to left-right positions in space), it seems highly unlikely that participants in the other tasks (where the left-right judgment was implicit) were aware that handedness was of interest. Presumably, the spatial manipulation in these questionnaires was invisible to participants, since it is perfectly ordinary for items on a questionnaire to be arranged in rows and columns; this is not usually because their relative right-left position is of veiled importance.

Among the minority of participants in these studies who were debriefed orally by the experimenter, not one mentioned handedness as an explanation for their placement of the good and bad animals in the diagram task, and not one mentioned handedness or spatial position as a reason for selecting one Fribble or verbal description over another. Participants in the Fribbles, shopping, and job tasks tended to justify their answers instead by referring to characteristics of the items within a pair. One participant said that a certain Fribble looked more intelligent because his antennae were pointing upward; another judged one applicant for the programmer job superior because he programmed in Python. In reality, due to counterbalancing, the differences between items within a pair cannot account for the body-specific pattern of data. Rather, spatial position resulted in body-specific judgments, perhaps by encouraging participants to attend selectively to positive features of the items located on their "good" side and to negative features of items on their "bad" side.

\section{Consequences of Body-Specific Representations of Good and Bad}

Do body-specific mental metaphors influence judgments outside of the laboratory? When choosing between two brands of cereal on the supermarket shelf, are we biased to buy the one on our dominant side? Does the placement of candidates' names on the left or right side of a ballot influence how likely they are to get elected? Is it a problem for liberal parties that they are on the left of the political spectrum, but most voters are right-handed? It may seem implausible that the incidental association of products or people with locations in physical space could influence our judgments about them, but as Wilson and Nisbett (1978) demonstrated, our intuitions about the factors that shape our judgments can be misleading. Some judgments may be impervious to the influence of irrelevant spatial factors. Optimistically, many of our decisions, from what to buy to whom we should vote for, are anchored in firm opinions developed after extensive research or prior experience. Yet, other routine decisions are at least partly impulsive, underinformed, or rooted in gut feelings rather than facts (e.g., Ambady \& Rule, 2007; Oppenheimer, 2008). How much of the variance in these judgments might be accounted for by the implicit influence of mental metaphors?

Even subtle influences of spatial location could have measurable real-world consequences. Because right-handed participants outnumbered left-handers in Experiments 1-5, a significantly greater number of participants showed a Good Is Right bias (53\%) than a 
Good Is Left bias (47\%), overall, collapsing across right- and left-handers $\left(p_{\text {rep }}=0.97\right)$. This $3 \%$ difference might seem negligible, but political elections are routinely won and lost by much slimmer margins. Even for presidential elections, votes have been shown to depend substantially on subtle, irrelevant factors like the order in which candidates' names were listed on the ballot (Krosnick, Miller, \& Tichy, 2004). The Fribbles, shopping, and job tasks are similar to tasks we perform in our daily lives: purchasing items found on the left or right side of a catalog or computer screen; ordering dishes from the left or right page of a restaurant menu; selecting candidates-for the Parent-Teacher Association or for political office-from the left or right side of a roster. Because right-handers outnumber left-handers in the general population, right-handers' mental metaphors should have a greater overall effect on the collective judgments made by knitting circles, neighborhoods, or nations. If we want to win approval, garner customers, or accumulate votes, the right side may be the right place to be.

\section{Conclusions}

Abstract concepts with positive and negative emotional valence are mentally represented, in part, via associative mappings from physical space (i.e., mental metaphors). This article documents a well-known mapping from vertical space to valence (Good Is Up, Bad Is Down), which is encoded in linguistic expressions and which is also evident in participants' nonlinguistic diagrams. In addition, it documents a new body-specific mapping from horizontal space to valence that is not found in any known languages or cultural artifacts but that corresponds to patterns of bodily experience. Whereas right-handers tend to associate right with positive ideas and left with negative ideas, left-handers show the opposite pattern, associating positive attributes like goodness, intelligence, attractiveness, and honesty with the left side of space. Both groups implicitly associate good things more strongly with their dominant side: the side on which they can act more fluently with their dominant hands. These results validate the bodyspecificity hypothesis. People with different kinds of bodies, who interact with their physical environments in systematically different ways, form correspondingly different mental representations, even in abstract domains.

Cultural conventions associating good with "right" and bad with "left" may reflect the implicit body-specific preferences of righthanders, who greatly outnumber left-handers. But this link between culture and body appears to be largely historical (at least for some cultures). The implicit mental metaphor Good Is Right does not appear to be universal, even though cultural conventions that link right with "good" may be. What appears to be universal is not a single mapping from space to valence but rather the propensity to represent ideas with positive and negative valence, in part, via body-specific associative links with our dominant and nondominant sides of space. As such, universal processes of correlational learning can give rise to body-specific mental representations.

Aside from the possible real-world implications of links between handedness and left-right preference, the discovery of bodyspecific mental metaphors for valence has several theoretical implications. Because patterns in language are tightly coupled with patterns of body-world interactions, previous studies have been unable to determine the extent to which mental metaphors from space to valence arise in the individual learner due to correlations in bodily experience or to correlations in linguistic experience. But framing experimental hypotheses in terms of body-specificity allowed the possible influences of language and culture to be distinguished from the influences of perceptuomotor experience. Linguistic and cultural experience cannot predict or explain the body-specific Good Is Left metaphor in left-handers or the difference between right- and left-handers' judgments. Thus, these data provide unequivocal evidence for an embodied origin of at least some abstract concepts. Like research on linguistic relativity and cultural relativity, research on what I will call by analogy bodily relativity can elucidate ways in which particular patterns of experience can give rise to corresponding habits of thinking, perceiving, and acting.

\section{References}

Ahern, G. L., \& Schwartz, G. E. (1979). Differential lateralization for positive versus negative emotion. Neuropsychologia, 17, 693-697.

Ambady, N., \& Rule, N. O. (2007). Thin slices of behavior. In R. F. Baumeister \& K. D. Vohs (Eds.), Encyclopedia of social psychology. Thousand Oaks, CA: Sage.

Barsalou, L. (1999). Perceptual symbol systems. Behavioral and Brain Sciences, 22, 577-609.

Barsalou, L., Niedenthal, P. M., Barbey, A. K., \& Ruppert, J. A. (2003). Social embodiment. In B. H. Ross (Ed.), The psychology of learning and motivation (pp. 43-92). New York: Academic Press.

Beilock, S. L., \& Holt, L. E. (2007). Embodied preference judgments: Can likeability be driven by the motor system? Psychological Science, 18, 51-57.

Boroditsky, L. (2000). Metaphoric structuring: Understanding time through spatial metaphors. Cognition, 75, 1-28.

Boroditsky, L. (2001). Does language shape thought? Mandarin and English speakers' conceptions of time. Cognitive Psychology, 43, 1-22.

Carey, S. (2004). Bootstrapping and the origins of concepts. Daedalus, 133(1), 59-68.

Casasanto, D. (2008a, November). The body-specificity hypothesis. Paper presented at the 49th Annual Meeting of the Psychonomic Society, Chicago.

Casasanto, D. (2008b). Similarity and proximity: When does close in space mean close in mind? Memory \& Cognition, 36, 1047-1056.

Casasanto, D. (2008c). Who's afraid of the big bad whorf: Crosslingusitic differences in temporal language and thought. Language Learning, 58(s1), 63-79.

Casasanto, D., \& Boroditsky, L. (2008). Time in the mind: Using space to think about time. Cognition, 106, 579-593.

Casasanto, D., \& Dijkstra, K. (2009). Motor action and emotional memory. Manuscript submitted for publication.

Casasanto, D., \& Nabieva, S. (2007, July). Automatic activation of spatial schemas for abstract concepts. Paper presented at the 10th International Cognitive Linguistics Association, Krakow, Poland.

Chatterjee, A. (2001). Language and space: Some interactions. Trends in Cognitive Science, 5, 55-61.

Clark, H. H. (1969). Linguistic processes in deductive reasoning. Psychological Review, 76, 387-404.

Clark, H. H. (1973). Space, time, semantics and the child. In T. E. Moore (ed.), Cognitive development and the acquisition of language (pp. 27 63). New York: Academic Press.

Corballis, M., \& Beale, I. (1976). Psychology of left and right. Hillsdale, NJ: Erlbaum.

Coren, S. (1992). The left-hander syndrome: The causes and consequences of left-handedness. New York: Random House.

Coren, S. (1993). Measurement of handedness via self-report: The rela- 
tionship between brief and extended inventories. Perceptual and Motor Skills, 73, 1035-1042.

Cretenet, J., \& Dru, V. S. (2004). The influence of unilateral and bilateral arm flexion vs. extension on judgments: An exploratory case of a motor congruence hypothesis. Emotion, 4, 282-294.

Damasio, A. (2001, October 25). Fundamental feelings. Nature, 413, 781.

Davidson, R. J. (1992). Anterior cerebral asymmetry and the nature of emotion. Brain and Cognition, 20, 125-151.

Davidson, R. J., Ekman, P., Saron, C. D., Senulis, J. A., \& Friesen, W. V. S. (1990). Approach-withdrawal and cerebral asymmetry: Emotional expression and brain physiology I. Journal of Personality and Social Psychology, 58, 330-341.

Davidson, R. J., \& Fox, N. A. (1982, December 17). Asymmetrical brain activity discriminates between positive and negative affective stimuli in human infants. Science, 218, 1235-1237.

Dehaene, S., Bossini, S., \& Giraux, P. (1993). The mental representation of parity and number magnitude. Journal of Experimental Psychology: General, 122, 371-396.

Dimond, S. J., Farrington, L., \& Johnson, P. (1976, June 24). Differing emotional response from right and left hemispheres. Nature, 261, 690692

Evans, V. S. (2004). The structure of time: Language, meaning and temporal cognition. Amsterdam: John Benjamins.

Everhart, D. E., Harrison, D. W., \& Crews, W. D., Jr. (1996). Hemispheric asymmetry as a function of handedness: Perception of facial affect stimuli. Perceptual and Motor Skills, 82(1), 264-266.

Feldman, J. (2006). From molecules to metaphor: A neural theory of language. Cambridge, MA: MIT Press.

Gallese, V. S., \& Lakoff, G. (2005). The brain's concepts: The role of the sensory-motor system in conceptual knowledge. Cognitive Neuropsychology, 22, 455-479.

Gentner, D., Bowdle, B., Wolff, P., \& Boronat, C. (2001). Metaphor is like analogy. In D. Gentner, K. J. Holyoak, \& B. N. Kokinov (Eds.), The analogical mind: Perspectives from cognitive science (pp. 199-253). Cambridge, MA: MIT Press.

Gentner, D., Brem, S., Ferguson, R. W., Markman, A. B., Levidow, B. B., Wolff, P., \& Forbus, K. D. (1997). Analogical reasoning and conceptual change: A case study of Johannes Kepler. Journal of the Learning Sciences, 6(1), 3-40.

Goldstone, R., \& Barsalou, L. (1998). Reuniting perception and conception. Cognition, 65, 231-262.

Goodglass, H., \& Quadfasel, F. (1954). Language laterality in left-handed aphasics. Brain, 4, 521-548.

Gould, G. M. (1908). Righthandedness and lefthandedness. Philadelphia: Lippincott.

Hebb, D. O. (1949). The organization of behavior. New York: Wiley.

Kövecses, Z. (2005). Metaphor in culture. Universality and variation. Cambridge, England: Cambridge University Press.

Keysar, B., \& Bly, B. (1995). Intuitions of the transparency of idioms: Can one keep a secret by spilling the beans? Journal of Memory and Language, 34, 89-109.

Killeen, P. R. (2005) An alternative to null hypothesis significance tests. Psychological Science, 16, 345-353.

Killgore, W. D. S., \& Yurgelun-Todd, D. A. (2007). The right-hemisphere and valence hypotheses: Could they both be right (and sometimes left)? Social Cognitive and Affective Neuroscience, 2, 240-250.

Kita, S., \& Essegbey, J. (2001). Pointing left in Ghana: How a taboo on the use of the left hand influences gestural practices. Gesture, 1(1), 73-95.

Krosnick, J. A., Miller, J. M., \& Tichy, M. P. (2004). An unrecognized need for ballot reform: Effects of candidate name order. In A. N. Crigler, M. R. Just, \& E. J. McCaffery (Eds.), Rethinking the vote: The politics and prospects of American election reform. New York: Oxford University Press.
Lakoff, G., \& Johnson, M. (1980). The metaphorical structure of the human conceptual system. Cognitive Science, 4, 195-208.

Lakoff, G., \& Johnson, M. (1999). Philosophy in the flesh: The embodied mind and its challenge to Western thought. Chicago: University of Chicago Press.

Longcamp, M., Anton, J. L., Roth, M., \& Velay, J. L. (2005). Premotor activations in response to visually presented single letters depend on the hand used to write: A study on left-handers. Neuropsychologia, 43, $1801-1809$.

Maas, A., \& Russo, A. (2003). Directional bias in the mental representation of spatial events: Nature or culture? Psychological Science, 14, 296-301.

Machery, E. (2007). Concept empiricism: A methodological critique. Cognition, 104, 19-46.

Maxwell, J. S., \& Davidson, R. J. (2007). Emotion as motion: Asymmetries in approach and avoidant actions. Psychological Science, 18, 1113-1119.

McFarland, R. A., \& Kennison, R. (1989). Handedness affects emotional valence asymmetry. Perceptual and Motor Skills, 68, 435-441.

Meier, B. P., \& Robinson, M. D. (2004). Why the sunny side is up: Associations between affect and vertical position. Psychological Science, 15, 243-247.

Murphy, G. (1996). On metaphoric representation. Cognition, 60, 173-204.

Murphy, G. (1997). Reasons to doubt the present evidence for metaphoric representation. Cognition, 62, 99-108.

Natale, M., Gur, R. E., \& Gur, R. C. (1983). Hemispheric asymmetries in processing emotional expressions. Neuropsychologia, 19, 609-613.

Oldfield, R. C. (1971). The assessment and analysis of handedness: The Edinburgh Inventory. Neuropsychologia, 9, 97-113.

Oppenheimer, D. M. (2008). The secret life of fluency. Trends in Cognitive Science, 12(6), 237-241.

Piaget, J. (1927/1969). The child's conception of time. New York: Ballantine.

Pinker, S. (1997). How the mind works. New York: Norton.

Prinz, J. (2002). Furnishing the mind. Cambridge, MA: MIT Press.

Reber, R., Winkielman, P., \& Schwarz, N. (1998). Effects of perceptual fluency on affective judgments. Psychological Science, 9(1), 45-48.

Riskind, J. H., \& Gotay, C. C. (1982). Physical posture: Could it have regulatory or feedback effects on motivation and emotion? Motivation and Emotion, 6, 273-298.

Robinson, E. S. (1933). The psychology of public education. American Journal of Public Health, 23, 123-128.

Scharine, A. A., \& McBeath, M. K. (2002). Right-handers and Americans favor turning to the right. Human Factors, 44(1), 248-256.

Schiff, B. B., \& Bassel, C. (1996). Effects of asymmetrical hemispheric activation on approach and withdrawal responses. Neuropsychology, 10, $557-564$.

Schubert, T. (2005). Your highness: Vertical positions as perceptual symbols of power. Journal of Personality and Social Psychology, 89, 1-21.

Simmons, W. K., Ramjee, V., Beauchamp, M. S., McRae, K., Martin, A., \& Barsalou, L. W. (2007). A common neural substrate for perceiving and knowing about color. Neuropsychologia, 45, 2802-2810.

Stepper, S., \& Strack, F. (1993). Proprioceptive determinants of emotional and nonemotional feelings. Journal of Personality and Social Psychology, 64, 211-220.

Talmy, L. (1988). Force dynamics in language and cognition. Cognitive Science, 12, 49-100.

Tomasello, M. (2003). Constructing a language: A usage-based theory of acquisition. Cambridge, MA: Harvard University Press.

Tversky, B., Kugelmass, S., \& Winter, A. (1991). Cross-cultural and developmental trends in graphic productions. Cognitive Psychology, 23, 515-557.

Van den Bergh, O., Vrana, S., \& Eelen, P. (1990). Letters from the heart: Affective categorization of letter combinations in typists and nontypists. Journal of Experimental Psychology: Learning, Memory, and Cognition, 16, 1153-1161. 
Vygotsky, L. (1934/1986). Thought and language. Cambridge, MA: MIT Press.

Willems, R. W., Hagoort, P., \& Casasanto, D. (in press). Body-specific representations of action verbs: Neural evidence from right- and lefthanders. Psychological Science.

Williams, L. E., \& Bargh, J. A. (2008). Keeping one's distance: The influence of spatial distance cues on affect and evaluation. Psychological Science, 19, 302-308.

Williams, S. M. (1986). Factor analysis of the Edinburgh Handedness Inventory. Cortex, 22, 325-326.

Wilson, T. D., \& Nisbett, R. E. (1978). The accuracy of verbal reports about the effects of stimuli on evaluations and behavior. Social Psychology, 41, 118-131.
Zebian, S. (2005). Linkages between number concepts, spatial thinking, and directionality of writing: The SNARC effect and the reverse SNARC effect in English and Arabic monoliterates, biliterates, and illiterate Arabic speakers. Journal of Cognition \& Culture, 5, 165-190.

Zwaan, R. A., \& Yaxley, R. H. (2003a). Hemispheric differences in semantic-relatedness judgments. Cognition, 87, B79-B86.

Zwaan, R., \& Yaxley, R. H. (2003b). Spatial iconicity affects semanticrelatedness judgments. Psychonomic Bulletin \& Review, 10, 954-958.

Received October 12, 2008

Revision received February 18, 2009

Accepted February 18, 2009

\section{Call for Nominations}

The Publications and Communications (P\&C) Board of the American Psychological Association has opened nominations for the editorships of Experimental and Clinical Psychopharmacology, Journal of Abnormal Psychology, Journal of Comparative Psychology, Journal of Counseling Psychology, Journal of Experimental Psychology: Human Perception and Performance, Journal of Personality and Social Psychology: Attitudes and Social Cognition, PsycCRITIQUES, and Rehabilitation Psychology for the years 2012-2017. Nancy K. Mello, PhD, David Watson, PhD, Gordon M. Burghardt, PhD, Brent S. Mallinckrodt, PhD, Glyn W. Humphreys, PhD, Charles M. Judd, PhD, Danny Wedding, PhD, and Timothy R. Elliott, PhD, respectively, are the incumbent editors.

Candidates should be members of APA and should be available to start receiving manuscripts in early 2011 to prepare for issues published in 2012. Please note that the P\&C Board encourages participation by members of underrepresented groups in the publication process and would particularly welcome such nominees. Self-nominations are also encouraged.

Search chairs have been appointed as follows:

- Experimental and Clinical Psychopharmacology, William Howell, PhD

- Journal of Abnormal Psychology, Norman Abeles, PhD

- Journal of Comparative Psychology, John Disterhoft, PhD

- Journal of Counseling Psychology, Neil Schmitt, PhD

- Journal of Experimental Psychology: Human Perception and Performance, Leah Light, $\mathrm{PhD}$

- Journal of Personality and Social Psychology: Attitudes and Social Cognition, Jennifer Crocker, $\mathrm{PhD}$

- PsycCRITIQUES, Valerie Reyna, PhD

- Rehabilitation Psychology, Bob Frank, PhD

Candidates should be nominated by accessing APA's EditorQuest site on the Web. Using your Web browser, go to http://editorquest.apa.org. On the Home menu on the left, find "Guests." Next, click on the link "Submit a Nomination," enter your nominee's information, and click "Submit."

Prepared statements of one page or less in support of a nominee can also be submitted by e-mail to Emnet Tesfaye, P\&C Board Search Liaison, at emnet@apa.org.

Deadline for accepting nominations is January 10, 2010, when reviews will begin. 